\title{
Die Plattform Schweizerisches Rettungswesen der FMH
}

Die Plattform Schweizerisches Rettungswesen der FMH, an der die Vertreter aller am Rettungswesen beteiligten und interessierten Fachgesellschaften teilnehmen, hat kürzlich im Zusammenhang mit der Beschreibung der Qualifikationen der in der Rettungskette integrierten Fachkräfte die Qualitätskriterien des ärztlichen Notfalldienstes verabschiedet. Sie sollen als Richtschnur für die zukünftige Gestaltung des ärztlichen Notfalldienstes im Sinne der Qualitätsförderung dienen. Sie sind gleichzeitig eine Deklaration gegenüber unseren Partnern im Rettungswesen, aber auch gegenüber Behörden und Versicherern.

Die Plattform hat bewusst auf Detailvorgaben verzichtet. Damit soll sichergestellt bleiben, dass die Kantonalen Ärztegesellschaften den Notfalldienst auch weiterhin den infrastrukturellen, topographischen und personellen Gegebenheiten in ihrem Verantwortungsbereich angepasst betreiben können. Den Fachgesellschaften andererseits bleibt damit die nötige Flexibilität in der Gestaltung der notfallmedizinischen Kurse erhalten.

Auf vielfachen Wunsch ist die Plattform daran, eine Standard-Ausrüstungsliste für den Dienstarzt zu erstellen und als Anhang zu den Qualitätskriterien herauszugeben. Auch diese Liste ist als nützliche Richtschnur zu verstehen und kann den besonderen Gegebenheiten angepasst werden. Sie wird zurzeit den jüngsten Empfehlungen der American Heart Association angepasst und demnächst separat veröffentlicht.

Dr. med. Reto Laetsch, Vorsitzender

Plattform Schweizerisches Rettungswesen der FMH 